\title{
Delay in the Recovery of Normal Sleep-Wake Cycle after Disruption of the Light-Dark Cycle in Mice: A Bipolar Disorder-Prone Animal Model?
}

\author{
Sun Hwa Jung ${ }^{1}$, Je-Min Park ${ }^{2}$, Eunsoo Moon${ }^{1}$, Young In Chung ${ }^{2}$, Byung Dae Lee', \\ Young Min Lee ${ }^{1}$, Ji Hoon Kim², Soo Yeon Kim¹, and Hee Jeong Jeong ${ }^{1}$ \\ ${ }^{1}$ Department of Psychiatry, Pusan National University Hospital, Busan, Republic of Korea \\ ${ }^{2}$ Department of Psychiatry, Pusan National University School of Medicine, Busan, Republic of Korea
}

Objective Disruption of the circadian rhythm is known as a provoking factor for manic episodes. Individual differences exist in the recovery rate from disruption in the general population. To develop a screening method to detect individuals vulnerable to bipolar disorder, the authors observed the relationship between the recovery of the normal sleep-wake cycle after switching the light-dark (LD) cycle and quinpirole-induced hyperactivity in mice.

Methods Sixteen male mice (age of 5 weeks, weight 28-29 gm) were subjected to a circadian rhythm disruption protocol. Sleep-wake behaviors were checked every 5 min for a total duration of 15 days, i.e., 2 days of baseline observations, 3 days of LD cycle changes, and 10 days of recovery. During the dark cycle on the 16th experimental day, their general locomotor activities were measured in an open field for 120 minutes after an injection of quinpirole $(0.5 \mathrm{mg} / \mathrm{kg}$, s.c.).

Results The individual differences in the recovery rate of the baseline sleep-wake cycle were noted after 3 days of switching the LD cycle. Fifty percent $(n=8)$ of the mice returned to the baseline cycle within 6 days after normalizing the LD cycle (early recovery group). The locomotor activities of mice that failed to recover within 6 days (delayed recovery group) were significantly higher (mean rank= 12.25) than those of the early recovery group (mean rank=4.75, $\mathrm{u}=62.0, \mathrm{p}=0.001$, Mann-Whitney $\mathrm{U}$ test).

Conclusion Given that the quinpirole-induced hyperactivity is an animal model of bipolar disorder, our results suggest individuals who have difficulties in recovery from circadian rhythm disruption may be vulnerable to bipolar disorder. Psychiatry Investig 2014;11(4):487-491

Key Words Bipolar disorder, Circadian rhythm, Quinpirole, Locomotor activity, Open field test, Animal model.

\section{INTRODUCTION}

Bipolar disorder, characterized by manic and depressive episodes, is known as a chronic and recurrent disease. ${ }^{1}$ Bipolar disorder can be triggered by a variety of biological, psychosocial, and genetic factors. Sleep disruption caused by transmeridian travel and sleep loss have been demonstrated as one of the important factors associated with the pathogenesis of bipolar disorder. ${ }^{2,3}$ In addition, bipolar patients have

Received: June 24, 2013 Revised: August 16, 2013

Accepted: September 30, 2013 Available online: October 20, 2014

$\triangle$ Correspondence: Je-Min Park, MD, PhD

Department of Psychiatry, Pusan National University School of Medicine, 179 Gudeok-ro, Seo-gu, Busan 602-739, Republic of Korea

Tel: +82-51-240-7307, Fax: +82-51-248-3648, E-mail: jmback@pusan.ac.kr

(a) This is an Open Access article distributed under the terms of the Creative Commons Attribution Non-Commercial License (http://creativecommons.org/licenses/bync/3.0) which permits unrestricted non-commercial use, distribution, and reproduction in any medium, provided the original work is properly cited. reported abnormal sleep patterns such as phase advance, phase delay and altered total sleep duration during remitted state as well as symptomatic periods. ${ }^{4-8}$

Individual differences exist in the recovery rate after disruption of the circadian rhythm. ${ }^{7-10}$ Life events such as traveling across several time zones and shift work schedules can disturb the circadian clock and lead to fatigue, insomnia and irritability. These symptoms such as jet lag are provoked by transients generated during the resetting of the internal pacemaker to re-synchronize with the external time. The individual differences in tolerance to the sleep deprivation from jet $\operatorname{lag}^{7}$ and in adaptation to shift work schedules have been reported in the general population. ${ }^{8}$ In rodents, these differences could be observed by an abrupt change in the lighting schedule. Mice needed about 4-6 days to recover to their normal sleep-wake cycle after the light-dark cycle disruption. ${ }^{9}$ 
Bipolar patients may have genetic vulnerability in the regulation of circadian rhythms and the sleep-wake cycle. There has been a considerable amount of evidence on circadian rhythm dysfunction among patients with bipolar disorder. ${ }^{6,10,11}$ Circadian activity patterns of the bipolar patients were reported to have less stable and more variable than their control counterparts. ${ }^{6}$ Seventy percent of the bipolar disorder patients in euthymic state exhibited clinically significant sleep disturbance compared to patients with insomnia or normal controls. ${ }^{11}$ Clock gene polymorphism may contribute to instability of sleep-wake cycle and vulnerabilities to bipolar disorder. ${ }^{10}$ However, it is undetermined yet if individuals who have difficulties in adapting to circadian rhythm disruption would be vulnerable to bipolar disorder.

Behavioral changes in the animal models of mania can present two basic and distinct dimensions: subjective and objective aspects. Subjective changes include modulation of emotions such as fear, aggressiveness, irritability, euphoria and dysphoria. Objective changes basically include aspects related to the locomotor activities which can be measured by the open field test. ${ }^{12}$ Meanwhile, amphetamines and other psychostimulants, including cocaine and some dopaminergic agonists, such as quinpirole, can induce locomotor hyperactivity in animals and are used as pharmacological animal models of bipolar disorder. ${ }^{12}$ To develop a screening method to detect individuals vulnerable to bipolar disorder, the authors observed the relationship between the recovery of the normal sleep-wake cycle after switching the light-dark cycle and quinpirole-induced hyperactivity in mice.

\section{METHODS}

\section{Animals}

Sixteen male mice (age of 5 weeks, weight 28-29 gm) from ICR (Institute of Cancer Research, Pusan National University Hospital, Republic of Korea) were used in this study. All mice were housed in individual cages under a 12 hour artificial light-dark (LD) cycle at a constant temperature of $20 \pm 1^{\circ} \mathrm{C}$ and relative humidity of $40-60 \%$. Food and water were provided ad libitum. Mice were entrained to a standard LD cycle for at least one week prior to the start of the experiments. The experimental procedures and protocols used in this study were reviewed and approved by the Ethics Committee on Animal Research at Pusan National University.

\section{Circadian rhythm disruption procedure and sleep-wake measurements}

Circadian rhythm disruption was done by shifting the 12 hour lighting condition. After one week of entrainment to LD conditions, mice were under the standard LD cycle for two more days to record their baseline sleep-wake cycles. On the 3rd experimental day, the LD cycle was abruptly shifted to the dark-light cycle (DL, lights on from 8 P.M. to 8 A.M., disruption phase). On the 6th experimental day, mice were re-entrained to the standard LD cycle (recovery phase).

Sleep-wake behaviors were videotaped for a total of 15 days, i.e. 2 days of baseline, 3 days of changed light-dark cycle, and 10 days of recovery. Mice were visually scored for their sleep-wake activities at 5 minute intervals (sleep: 1, wake: 0 ). These values were summed and hourly percentages of sleep-wake were determined. The sleep state is marked by several easily observed behaviors, including adoption of species-specific sleep postures with the eye closed. ${ }^{13}$ Thus we scored an animal as having been behaviorally asleep only if its eyes were closed and either laying on its side or sitting curled up with the head tucked into the body without any movement other than very light and brief transitional changes in posture. This type of strategy has been previously used to assess the basic temporal distribution of behavioral sleep across the $24 \mathrm{hr}$ period. ${ }^{14}$

The normalization of sleep-wake pattern was defined by the time that sleep duration in light phase was higher than that in dark phase, similar pattern of first 2 days (baseline cycle), and the recovered pattern was consecutively continued, based on the comparison between the behavioral activity levels in dark and light phases.

\section{Quinpirole-induced locomotor activity measurements}

Quinpirole was diluted in deionized water and injected (S.C., $0.5 \mathrm{mg} / \mathrm{kg}$ ) under the nape of the neck during the dark period (from 8:00 P.M. to 9:00 P.M.) on the 16th experimental day. Their general locomotor activities were measured in an open field for 120 minutes after injection of quinpirole. The open field consisted of a square arena $(50 \times 50 \times 30 \mathrm{~cm}$, $1 \times \mathrm{w} \times \mathrm{h})$ made of an acrylic board. The arena was divided into 25 squares $(10 \times 10 \mathrm{~cm})$. Immediately after injection, a mouse was placed at the center of the arena and videotaped for 120 minutes. We counted the number of lines crossed by its front leg as a measurement of locomotor activity.

\section{Statistical analysis}

The sleep patterns of the light-dark cycle for 15 days are shown in Figure 1. Every sleep-wake pattern was compared to reveal the moment at which fifty percent of the mice returned to the baseline cycle. Fifty percent $(n=8)$ of the mice returned to the baseline cycle in 6 days after normalization of the light-dark cycle, and they were divided into two groups according to the total sleep duration of dark period on the 6th day after normalizing the light-dark cycle. Considering 


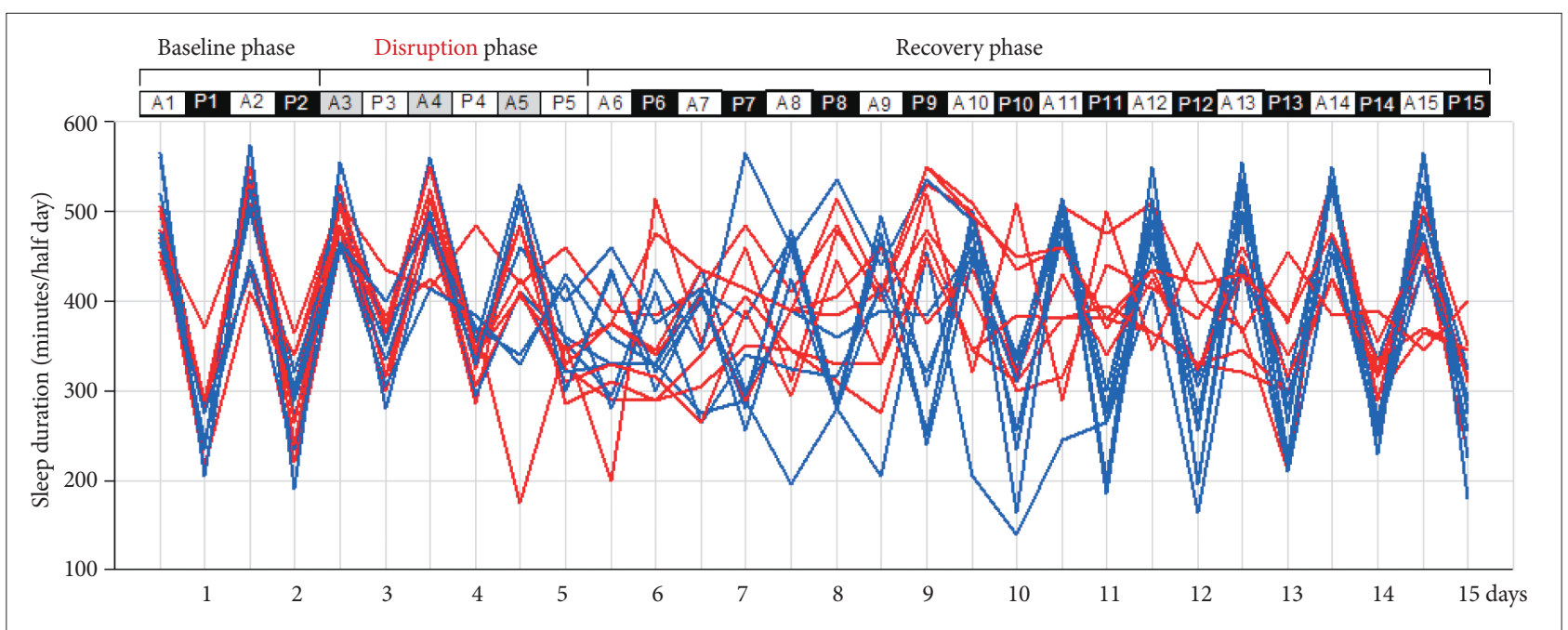

Figure 1. Sleep-wake patterns observed by a circadian rhythm disruption protocol. White bar: light cycle, Black bar: dark cycle, Gray bar: dark cycle during disruption phase. A: from 8:00 A.M. to 8:00 P.M., P: from 8:00 P.M. to 8:00 A.M. of the day after. Blue line: early recovery group, Red line: delayed recovery group.

that mice are nocturnal animals, we named the lower $50 \%$ group as the early recovery group ( $\mathrm{n}=8)$ and the upper $50 \%$ group as the delayed recovery group $(\mathrm{n}=8)$. Mann-Whitney U test was used to compare the distribution of locomotor activities for 120 minutes. All statistical analyses were performed using the Statistical Package for Social Science for Windows, version 18.0 and the level of statistical significance was set at $\mathrm{p}<0.05$.

\section{RESULTS}

Figure 1 shows individual total sleep durations of the lightdark period for 15 days, i.e. baseline phase, circadian rhythm disruption phase, and recovery phase. Table 1 shows total sleep duration (minutes/half day) of each mouse during light-dark period. There were individual differences in the recovery rate of baseline sleep-wake cycle after 3 days of light-dark cycle switching.

Fifty percent $(\mathrm{n}=8)$ of the mice returned to the baseline cycle in 6 days after normalizing the light-dark cycle (early recovery group). Locomotor activities of mice that failed to recover in 6 days (delayed recovery group) were significantly higher (mean $\pm \mathrm{SD}=846.9 \pm 335.1$, mean $\mathrm{rank}=12.25$ ) than those of the early recovery group (mean $\pm S D=265.5 \pm 44.8$, mean rank=4.75; Mann-Whitney $\mathrm{U}$ test, $\mathrm{u}=62.0, \mathrm{p}=0.001$ ) (Figure 2).

\section{DISCUSSION}

In this study, we found that fifty percent of the mice returned to the baseline cycle in 6 days after normalizing the light-dark cycle, and this result is consistent with the results of previous studies. One report demonstrated that mice need around 4-6 days to recover their normal sleep-wake cycle after light-dark cycle disruption. ${ }^{9}$ Another study noted that 5-6 days were required to recover the normal sleep-wake cycle in mice after a $6 \mathrm{hr}$ advance in the lighting schedule. ${ }^{15}$ In the present study, there were some mice which could not recover their normal sleep-wake cycle during 10 days of recovery. Several studies have also suggested that there would be individual differences in the recovery rate from disruption. ${ }^{7,8} \mathrm{Fu}-$ ture research is expected to improve insight into the pathophysiology of mice showing delayed recovery.

We observed a relation between the recovery of the normal sleep-wake cycle after switching the light-dark cycle and quinpirole-induced hyperactivity in mice. We were able to demonstrate that locomotor activities of mice that failed to recover in 6 days (delayed recovery group) were significantly higher than those of the early recovery group. Several studies have suggested that circadian rhythm dysfunction can trigger mood episodes in bipolar disorder. There has been a considerable amount of evidence that sleep disturbance is by far the most commonly reported prodromal symptom prior to a manic episode. ${ }^{16}$ An improvement in mood was observed in $60 \%$ of depressed bipolar patients after total or partial sleep deprivation. ${ }^{17}$ Twenty nine percent of bipolar depressed patients became hypomanic or manic after one night of total sleep deprivation..$^{18}$ In addition, obstructive sleep apnea, in which sleep is disrupted by intermittent obstruction of the upper airway during which leads to repetitive brief arousals, may contribute to mania or treatment resistance. ${ }^{19}$ All triggers of mania, including biological causes (drug, hormones, etc.), psychic effects (separation, bereavement, etc.), and direct disturbance of sleep schedules (shift work, travel, etc.), 


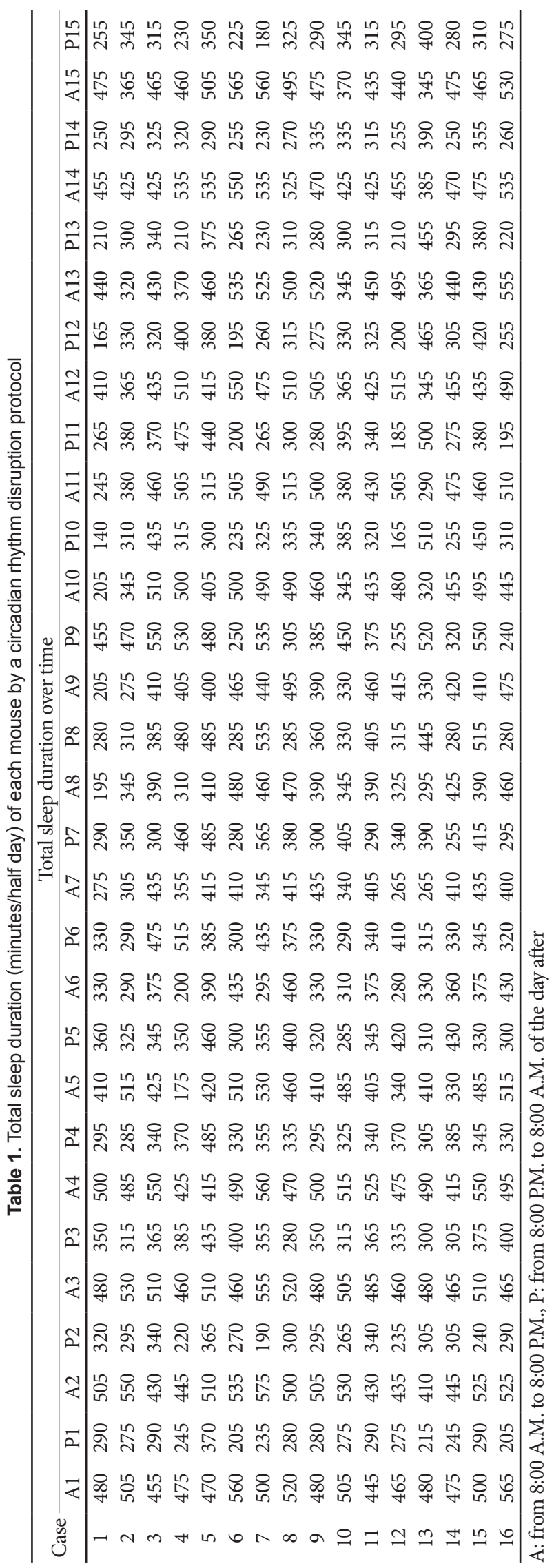

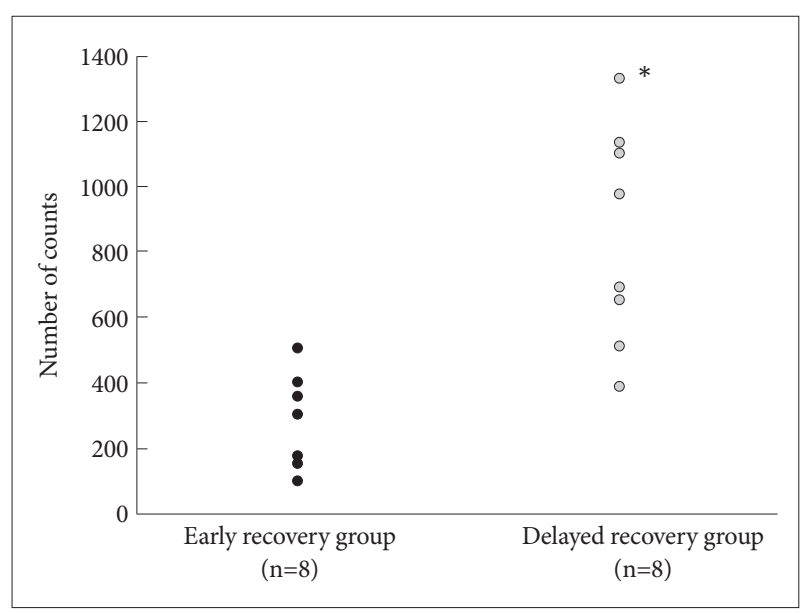

Figure 2. Locomotor activities in the open field test after quinpirole injection. Locomotor activities of mice failed to recover in 6 days (delayed recovery group) were significantly higher (mean \pm $S D=846.9 \pm 335.1$, mean rank=12.25) than those of the early recovery group (mean $\pm S D=265.5 \pm 44.8$, mean rank=4.75). *MannWhitney U test, $u=62.0, p=0.001$, two-tailed.

could be related to the genesis of mania through sleep reduction. $^{20}$

It was difficult to find out the objective screening method to detect the exact recovery timing of the normal sleep-wake cycle after switching the light-dark cycle. Moreover, we found little research which observed the sleep recovery phase. Loh et al. ${ }^{21}$ used a protocol similar to this study to estimate the distribution of behavioral sleep-wake before and after a $6 \mathrm{hr}$ advance. They assessed the basic temporal distribution of sleep across the $24 \mathrm{hr}$ period but did not assessed the sleep recovery phase.

Quinpirole, a D (dopamine)2/D3 agonist, induces a biphasic locomotor response by starting with inhibition followed by excitation, resembling the oscillating nature of bipolar disorder and showing face validity as an animal model of bipolar behavior. ${ }^{12}$ In particular, an acute injection of quinpirole induces locomotor excitation at higher doses above 0.5 $\mathrm{mg} / \mathrm{kg}{ }^{22}$ In mice, injection of amphetamine, cocaine, and dopamine agonists causes motor hyperactivity, as a maniclike symptom, which has been associated with the activation of the mesolimbic dopaminergic system originating from the ventral tegmental area to the nucleus accumbens. ${ }^{23}$

Considering that bipolar patients exhibit significantly more exploratory behaviors than healthy comparison subjects, ${ }^{24}$ and that dopamine is involved in the pathophysiology of mania, ${ }^{25}$ we presumed that individuals vulnerable to bipolar disorder would show an increase of quinpirole-induced hyperactivity. Fifty percent $(n=8)$ of the mice returned to the baseline cycle in 6 days after normalizing the light-dark cycle (early recovery group). Locomotor activities of mice that failed to recover in 6 days (delayed recovery group) were sig- 
nificantly higher than those of the early recovery group. The results showed that the delayed recovery group could be more vulnerable to bipolar disorder.

Given that quinpirole-induced hyperactivity is an animal model of bipolar disorder, our results suggest that individuals who have difficulties in adaptation to circadian rhythm disruption may be vulnerable to bipolar disorder. Further clinical research is needed to validate our results.

\section{Acknowledgments}

The results of this study were presented at the 2nd Congress of Asian College of Neuropsychophamacology, 23-24 September 2011, Seoul, Republic of Korea.

\section{REFERENCES}

1. Bebbington P, Ramana R. The epidemiology of bipolar affective disorder. Soc Psychiatry Psychiatr Epidemiol 1995;30:279-292.

2. Jauhar P, Weller MP. Psychiatric morbidity and time zone changes: a study of patients from Heathrow airport. Br J Psychiatry 1982;140:231235.

3. Young DM. Psychiatric morbidity in travelers to Honolulu, Hawaii. Compr Psychiatry 1995;36:224-228.

4. Winkler D, Pjrek E, Praschak-Rieder N, Willeit M, Pezawas L, Konstantinidis A, et al. Actigraphy in patients with seasonal affective disorder and healthy control subjects treated with light therapy. Biol Psychiatry 2005;58:331-336.

5. Millar A, Espie CA, Scott J. The sleep of remitted bipolar outpatients: a controlled naturalistic study using actigraphy. J Affect Disord 2004;80: 145-153.

6. Jones SH, Hare DJ, Evershed K. Actigraphic assessment of circadian activity and sleep patterns in bipolar disorder. Bipolar Disord 2005;7: 176-186.

7. Redfern P, Minors D, Waterhouse J. Circadian rhythms, jet lag, and chronobiotics: an overview. Chronobiol Int 1994;11:253-265.

8. Harma M. Sleepiness and shiftwork: individual differences. J Sleep Res 1995;4(S2):57-61.

9. Corinne J, Isabelle S, Gabriele H, Henrik O, Urs A. A guideline for analyzing circadian wheel-running behavior in rodents under different lighting conditions. Biol Proced Online 2005;7:101-116.

10. Mansour HA, Monk TH, Nimgaonkar VL. Circadian genes and bipolar disorder. Ann Med 2005;37:196-205.
11. Harvey AG, Schmidt DA, Scarna A, Semler CN, Goodwin GM. Sleeprelated functioning in euthymic patients with bipolar disorder, patients with insomnia, and subjects without sleep problems. Am J Psychiatry 2005;162:50-57.

12. Shaldubina A, Einat H, Szechtman H, Shimon H, Belmaker RH. Preliminary evaluation of oral anticonvulsant treatment in the quinpirole model of bipolar disorder. J Neural Transm 2002;109:433-440.

13. Campbell SS, Tobler I. Animal sleep: a review of sleep duration across phylogeny. Neurosci Biobehav Rev 1984;8:269-300.

14. Schwartz MD, Smale L. Individual differences in rhythms of behavioral sleep and its neural substrates in Nile grass rats. J Biol Rhythms 2005;20:526-537.

15. Reddy AB, Field MD, Maywood ES, Hastings MH. Differential resynchronisation of circadian clock gene expression within the suprachiasmatic nuclei of mice subjected to experimental jet lag. J Neurosci 2002; 22:7326-7330.

16. Jackson A, Cavanagh J, Scott J. A systematic review of manic and depressive prodromes. J Affect Disord 2003;74:209-217.

17. Wirz-Justice A, Van den Hoofdakker RH. Sleep deprivation in depression: what do we know, where do we go? Biol Psychiatry 1999;46:445453.

18. Wu JC, Bunney WE. The biological basis of an antidepressant response to sleep deprivation and relapse: review and hypothesis. Am J Psychiatry 1990;147:14-21.

19. Strakowski SM, Hudson JI, Keck PE Jr, Wilson DR, Frankenburg FR, Alpert JE, et al. Four cases of obstructive sleep apnea associated with treatment-resistant mania. J Clin Psychiatry 1991;52:156-158.

20. Wehr TA, Sack DA, Rosenthal NE. Sleep reduction as a final common pathway in the genesis of mania. Am J Psychiatry 1987;144:201-204.

21. Loh DH, Navarro J, Hagopian A, Wang LM, Deboer T, Colwell CS. Rapid Changes in the Light/Dark Cycle Disrupt Memory of Conditioned Fear in Mice. PLoS ONE 2010;5.

22. Eilam D, Szechtman H. Biphasic effect of D-2 agonist quinpirole on locomotion and movements. Eur J Pharmacol 1989;161:151-157.

23. Koob GF, Bloom FE. Cellular and molecular mechanisms of drug dependence. Science 1988;242:715-723.

24. Young JW, Minassian A, Paulus MP, Geyer MA, Perry W. A reversetranslational approach to bipolar disorder: rodent and human studies in the Behavioral Pattern Monitor. Neurosci Biobehav Rev 2007;31: 882-896.

25. Manji HK, Quiroz JA, Payne JL, Singh J, Lopes BP, Viegas JS, et al. The underlying neurobiology of bipolar disorder. World Psychiatry 2003;2: 136-146. 